\title{
Ontology-based representation and analysis of host-Brucella interactions
}

Yu Lin, Zuoshuang Xiang and Yongqun $\mathrm{He}^{*}$

\begin{abstract}
Background: Biomedical ontologies are representations of classes of entities in the biomedical domain and how these classes are related in computer- and human-interpretable formats. Ontologies support data standardization and exchange and provide a basis for computer-assisted automated reasoning. IDOBRU is an ontology in the domain of Brucella and brucellosis. Brucella is a Gram-negative intracellular bacterium that causes brucellosis, the most common zoonotic disease in the world. In this study, IDOBRU is used as a platform to model and analyze how the hosts, especially host macrophages, interact with virulent Brucella strains or live attenuated Brucella vaccine strains. Such a study allows us to better integrate and understand intricate Brucella pathogenesis and host immunity mechanisms.

Results: Different levels of host-Brucella interactions based on different host cell types and Brucella strains were first defined ontologically. Three important processes of virulent Brucella interacting with host macrophages were represented: Brucella entry into macrophage, intracellular trafficking, and intracellular replication. Two Brucella pathogenesis mechanisms were ontologically represented: Brucella Type IV secretion system that supports intracellular trafficking and replication, and Brucella erythritol metabolism that participates in Brucella intracellular survival and pathogenesis. The host cell death pathway is critical to the outcome of host-Brucella interactions. For better survival and replication, virulent Brucella prevents macrophage cell death. However, live attenuated B. abortus vaccine strain RB51 induces caspase-2-mediated proinflammatory cell death. Brucella-associated cell death processes are represented in IDOBRU. The gene and protein information of 432 manually annotated Brucella virulence factors were represented using the Ontology of Genes and Genomes (OGG) and Protein Ontology (PRO), respectively. Seven inference rules were defined to capture the knowledge of host-Brucella interactions and implemented in IDOBRU. Current IDOBRU includes 3611 ontology terms. SPARQL queries identified many results that are critical to the host-Brucella interactions. For example, out of 269 protein virulence factors related to macrophage-Brucella interactions, 81 are critical to Brucella intracellular replication inside macrophages. A SPARQL query also identified 11 biological processes important for Brucella virulence.
\end{abstract}

Conclusions: To systematically represent and analyze fundamental host-pathogen interaction mechanisms, we provided for the first time comprehensive ontological modeling of host-pathogen interactions using Brucella as the pathogen model. The methods and ontology representations used in our study are generic and can be broadened to study the interactions between hosts and other pathogens.

\footnotetext{
* Correspondence: yongqunh@med.umich.edu

Unit of Laboratory Animal Medicine, Department of Microbiology and Immunology, Center for Computational Medicine and Bioinformatics, and Comprehensive Cancer Center, University of Michigan Medical School, 1150 W. Medical Center Dr, Ann Arbor, Ml 48109, USA
}

(c) 2015 Lin et al. Open Access This article is distributed under the terms of the Creative Commons Attribution 4.0 International License (http://creativecommons.org/licenses/by/4.0/), which permits unrestricted use, distribution, and reproduction in any medium, provided you give appropriate credit to the original author(s) and the source, provide a link to the Creative Commons license, and indicate if changes were made. The Creative Commons Public Domain Dedication waiver (http://creativecommons.org/publicdomain/zero/1.0/) applies to the data made available in this article, unless otherwise stated. 


\section{Background}

In the field of infectious diseases, the study of the interactive relationships between pathogens and their hosts is critically important. An infectious disease is the result of intense interactions between a pathogen and its host. During these interactions both the host and the pathogen attempt to manipulate each other using a complex network mechanism to maximize their respective survival probabilities. For the goal of survival and replication, the pathogen may adapt different pathogenesis strategies to infect the host. On the other hand, the host may apply innate and adaptive immune defense mechanisms to fight against the invading pathogen. Different live attenuated vaccines may stimulate sufficient host immunity but does not induce damaging effects on the host. For decades, scientists have conducted research to study the different aspects of hostpathogen interactions. In order to obtain a full picture of the host-pathogen interaction mechanisms, separate data from those studies needs to be integrated. Thus, a strategy of knowledge representation, management and reasoning based on the huge data resources is in need. Such a strategy will enable the knowledge integration, complicated biological data analysis, and provide insights for biologists to generate new hypotheses.

Brucella is a Gram-negative, non-spore-forming, facultative, intracellular bacterium that causes chronic zoonotic brucellosis in humans and a variety of animal species [1]. Human brucellosis remains the most common zoonotic disease worldwide, with more than 500,000 new human cases reported annually [2]. A safe and effective human vaccine is required but does not yet exist. A rational vaccine design would benefit from insightful understanding of the interactions between host and Brucella, specifically, Brucella pathogenesis and host defense mechanisms. Brucella infections are typically chronic in nature [1], suggesting a continuous interaction between host and Brucella. To promote its long-term intracellular survival, Brucella minimizes the activation of host inflammatory mechanisms. For example, Brucella lipopolysaccharide (LPS) has 100 - to 1000 -fold decreased capability to activate proinflammatory TNF- $\alpha$ and IL-1 cytokines compared to similar concentrations of E. coli LPS [3]. Hundreds of Brucella protein virulence factors participate in the Brucella pathogenesis and interacting with host immune systems $[4,5]$. The immune systems in various Brucella hosts respond poorly against virulent Brucella strains but very well against live attenuated Brucella vaccine strains [6-8]. Such a complex host-pathogen interaction system involves a number of cells, molecules and biological processes. Therefore, the host-Brucella interactions present a good example for an ontology-based exploration of complex bacterial pathogenesis and host immunity mechanisms.

As an extension ontology of the Infectious Disease Ontology (IDO) [9], Brucellosis Ontology (IDOBRU) is developed previously at our lab [10]. Following the good practice of the OBO Foundry principles [11], IDOBRU was developed under the framework of the Basic Formal Ontology (BFO) [12] and IDO. BFO contains two branches, continuant and occurrent $[11,13]$. The continuant branch represents timeindependent entity such as material entity, and the occurrent branch represents time-related entity such as process. By aligning different domain ontologies under the two branches of BFO, the knowledge from broad biological areas could be captured and organized under a comprehensive ontology-level structure. Since IDO aligns with BFO, IDOBRU automatically aligns with BFO. IDOBRU exemplifies IDO in the case of brucellosis, which covers a broad range of topics, including host infection, zoonotic disease transmission, symptoms, virulence factors, pathogenesis, diagnosis, intentional release, vaccine prevention, and treatment [10].

Since the study of host-Brucella interactions has been a major brucellosis research effort, this paper goes beyond the simplified introduction of virulence factors and pathogenesis in the previous IDOBRU paper [10], and provides more detailed ontological representation on various aspects of the host-pathogen interactions. In this report, different types of the pathogen-side pathogenesis mechanisms and host-side immune defense strategies are described with specific examples. Comparing with the 245 virulence factors in the original IDOBRU ontology [10], current version includes 432 virulence factors in Brucella. Furthermore, a list of computer-understandable logic inference rules is defined in this study to make the virulence factors, host-Brucella interactions and related processes computable. Use cases are provided to demonstrate how such ontological representations and inference rule-based automated reasoning help data integration and query in the area of host-pathogen/vaccine interactions.

\section{Results}

In what follows, italics are used to make ontological axioms (i.e., statements that say what is true in the domain) (http://www.w3.org/TR/owl2-syntax/), simple bold words represent ontology relations, single quotes are used to represent ontology terms, and double quotes are used for text definitions or emphases.

\section{Overall design of ontological representing of host-Brucella interaction}

Various disciplines dissect interactions into different tailored meanings. The definition of an interaction in the host-pathogen interaction area includes a "two-way effect" (i.e., host's effect upon pathogen, and pathogen's effect upon host). The interactions between host (e.g., human) and Brucella exemplifies a host-pathogen interaction in the context of biology. As an intracellular bacterium, Brucella strains are able to invade, survive and replicate for 
prolonged periods within in vivo host cells or in vitro cultured host cells. The GO term 'interspecies interaction between organisms' (GO_0044419) is defined as "any process in which an organism has an effect on an organism of a different species". Without capturing the granularity at the host cell level, this GO term is not sufficient for our case. The definition of 'host-Brucella interaction' in IDOBRU is "an interspecies interaction that is the physical encounter of two parties: Brucella and its host organism or host cell. While interacting, Brucella and its host organism or host cell have effects upon each other." The interaction in this definition specifically emphasizes the physical encounter and interaction of the two parties in an action. Also, given that Brucella is an intracellular bacterium, it is important to explicitly mention both of the host organism and the host cell.

The host-Brucella interaction includes three main parts: 1) 'Brucella entry into host cell' (IDO_0101170) (the interaction at the interface between Brucella and host), 2) 'process of establishing Brucella infection in host' (IDO_0100426) (Brucella side response), and 3) 'host anti-Brucella process' (IDO_0100115) (host side response). The IDOBRU term 'Brucella entry into host cell' is a child term of GO 'entry of bacterium into host cell' (GO_0035635). The IDOBRU term 'process of establishing Brucella infection in host' is a child term of the IDO-core term 'process of establishing an infection' (IDO_0000603), which is under GO 'biological process'. The 'host anti-Brucella process' is a child term of GO 'biological process'.

As we mentioned before, IDOBRU adopted Basic Formal Ontology version 2 (BFO 2) as its top level ontology [10]. Favoring BFO is due to the integrative nature of IDOBRU, a representation of all aspects of brucellosis, as it requires integrating with other OBO library ontologies, including the Cell Type Ontology (CL) [14], Chemical Entities of Biological Interest (ChEBI) [15], Gene Ontology (GO) [16], Information Artifact Ontology (IAO) [17], Ontology of Biomedical Investigations [18], Ontology of General Medical Science (OGMS) (https://code.google. com/p/ogms/), Ontology of Genes and Genomes (OGG) [19], and Protein Ontology (PRO) [20]. BFO serves as a common structure and a formal framework to seamlessly integrate existing terms from all OBO foundry ontologies. Under this consideration, we adopt relations formally defined by the community as much as possible. To represent the relations between molecular entities and its related host-Brucella interactions, we adopted the relation has agent formally defined by Smith et al. [21]. In the definition provided by Smith et al., a $p$ has_agent $\mathrm{c}$ at $\mathrm{t}$ denotes a primitive relation between a process $p$, a continuant $c$ and a time $t$ at which the continuant $c$ is causally active in the process [21]. The notion of "causally active" is aimed to capture the "from-to" directionality nature of a biological process, which provides the explicit measure for the "two-way effect" interactions. Applying this relation to model host-Brucella interaction, we differentiate the host-Brucella interaction into two categories: 1) 'Brucella process towards host infection', and 2) host anti-Brucella process. The first category has Brucella as its agent, and the second category has a host as its agent. In another word, Brucella actively causes 'establishing Brucella infection in host', and host actively causes host anti-Brucella processes. The Brucella and host both play an agent role in the processes of host-Brucella interaction.

To go beyond the textual definitions and logically define the 'host-Brucella interaction' and many other ontology terms, two approaches were used. One approach was to use an ontological axiom(s). An ontological axiom is a statement that provides explicit logical assertions about three types of things: classes, individuals and properties (http://www.w3.org/TR/owl2-syntax/\#Axioms). The other facts implicitly contained in the ontology can be inferred using a reasoning software program (i.e., a reasoner). Another approach is based on inference rules. On the Semantic Web, the term "inference" means an automatic procedure that can generate new relationship(s) based on the data (e.g., ontology knowledge data) and some additional information in the form of a vocabulary, for example, a set of rules (http://www.w3.org/standards/ semanticweb/inference). An inference rule (IR) is a logical form consisting of a function that takes premises, analyzes the syntax, and returns a conclusion(s).

To improve data integration and discover new relationships and possible inconsistencies, we have defined seven inference rules (IRs) in this report. We have first developed inference rules to capture the physical interaction of the two entities (agents) in a host-Brucella interaction. We use the IF $p$ THEN $q$ inference rules to state that, given the truth of $p$, allows the truth of $q$ to be inferred. The IF...THEN rules are used as a knowledge representation format that can be easily understood and simple to implement by a computer [22]. Here we formalized the first inference rule (IR1) to define a host-Brucella interaction as following:

(IR1) IF ( $a$ agent_in $p, \cap b$ agent_in $p), \cap p$ is_a process, $\cap(a$ part_of $A, \cap b$ part_of $B) \cap(A$ is_a (host organism $\cup$ host cell), $\cap B$ is_a Brucella), THEN $p$ is_a 'host-Brucella interaction'

IR1 gives three constrains sufficient to define a direct host-Brucella interaction: 1) two entities $a$ and $b$ are agents in a process; 2 ) these two entities are parts of entity $A$ and $B$ respectively; 3$) A$ is a host organism or host cell, and $B$ is a Brucella bacterium. $A$ and $B$ are disjoint with each other. It is noted that while a direct host-Brucella interaction involves both host and Brucella molecules, a 
process that participates in the host-pathogen interaction may be just a typical host or pathogen process that is triggered by the direct host-Brucella interaction. In this article, we will provide many examples showing the pathogen-side or host-side processes following a direct host-Brucella interaction.

Besides IR1, this article will provide six other IF...THEN inference rules. The IR2 provides an inference that one process is preceded by another process after a direct hostBrucella interaction. IR3-IR7 is about the inference on a virulence factor. Different from ontology axioms that usually represent necessary or (necessary and sufficient) conditions, these IF...THEN inference rules represent sufficient criteria (IF conditions) for a specific inference (THEN conclusion).

While ontology axioms behave like inference rules, the Web Ontology Language (OWL) is unable to express all relations and inference rules (http://dior.ics.muni.cz/ $\sim$ makub/owl/\#ontology). One common language that can be used to define inference rules is the Semantic Web Rule Language (SWRL) developed based on a combination of the OWL language with the Rule Markup Language (http://www.w3.org/Submission/SWRL/). While inference rules do not have to be a part of the ontology, SWRL can be used to represent the rules in an ontology like IDOBRU as shown in Additional file 1. These inference rules described in an ontology have at least two functions. First, these rules in combination with a logic reasoner support ontology consistency check. If an ontology has errors in conflict with an inference rule, a reasoner like Hermit (http://hermit-reasoner.com/) will be able to detect the error. Second, an IF...THEN inference rule can generate a conclusion on an ontology instance based on specified conditions.

While inference rules are less frequently used in biomedical ontologies, the SPARQL Protocol and RDF Query Language (SPARQL) has been widely used for querying ontologies [23] and is familiar to the general readers in biomedical semantics. In this article, we have also provided many SPARQL examples to illustrate the usage of SPARQL in querying information related in host-Brucella interactions.

\section{Ontology modeling of different types of Brucella}

Brucella strains can be separated into smooth strains and rough Brucella strains depending on their lipopolysaccharide (LPS) compositions. As a major component of the outer membrane of Brucella, Brucella LPS is composed of three parts: $\mathrm{O}$ polysaccharide (or called O-chain or Oantigen), core oligosaccharide, and lipid A. The O polysaccharide, a repetitive glycan polymer attached to the core oligosaccharide, is the outermost domain of the LPS molecule. The presence or absence of the LPS O polysaccharide determines whether a Brucella strain has the smooth or rough phenotype, respectively. The presence of fulllength $\mathrm{O}$ polysaccharides would render the bacterium smooth, whereas the absence of $\mathrm{O}$ polysaccharides would make the bacterium rough [24]. Rough Brucella is usually attenuated, and it does not stimulate the production of anti-O polysaccharide antibody in an infected host. In contrast, most smooth Brucella strains are virulent, and they have intact $\mathrm{O}$ polysaccharides and can stimulate anti- $\mathrm{O}$ polysaccharide antibody in host [25]. Virulent wild type $B$. abortus, B. melitensis, B. neotomae, and B. suis are all smooth strains, and their rough strains (including many vaccine and vaccine candidate strains) are attenuated. However, virulent wild type $B$. canis and B. ovis are rough. Therefore, the virulent/attenuated and smooth/ rough characteristics of Brucella strains may not match each other.

In order to define the smooth and rough characteristics of Brucella, the negative statement of 'has no Brucella $\mathrm{O}$ polysaccharide' needs to be addressed. Ceusters et al. has given a set of 'lacks' relations to represent the negative findings in electronic health records [26]. For example, the relation lacks_part was defined in terms of the positive relation part_of, holds between a particular $p$ and a universal $u$ when $p$ has no $u$ as part [26]. However, all relations in OWL are relations between particulars by default and cannot represent the relation between a particular and a universal. It is possible to rely on the punning (an OWL2 feature) that allows OWL developers to use the URI of a class for an individual (http://www.w3.org/TR/owl2-newfeatures/\#F12:_Punning). Another shortcoming about the lacks_part relation in OWL is that the relation in OWL expression $C$ subClass $O f$ : lacks-part some $D$ implies the presence of an instance of $\mathrm{D}$ where the relation itself suggests the lack of such an instance [27]. Therefore, although initially we used the lacks_part relation, we have recently switched to the use of not has_part as suggested in the article [27]. Here we adopt their strategy to define the terms 'smooth Brucella strain' and 'rough Brucella strain' as follows:

Smooth Brucella strain = def a Brucella has_part some
'smooth Brucella lipopolysaccharide'
Rough Brucella strain= def $a$ Brucella has_part only
'rough Brucella lipopolysaccharide'
Smooth Brucella lipopolysaccharide = def a 'Brucella
lipopolysaccharide' has_part some 'Brucella O
polysaccharide'
Rough Brucella lipopolysaccharide = def a 'Brucella
lipopolysaccharide' not has_part some 'Brucella $O$
polysaccharide'

Ontology modeling of host-Brucella interaction subtypes Brucella bacteria are able to invade and infect both professional and non-professional phagocytes. The interactions 
between Brucella and these host cells dictate the outcomes of the infection [28]. At least three types of host cells are recognized: macrophages, dendritic cells, and epithelial cells (e.g., placental trophoblast cells) [28]. Some cell lines, such as mouse macrophage cell line J774 [25] and human epithelial cell line HeLa [29] are important models for studying host-Brucella interactions. Those cell types and cell lines have been imported into IDOBRU from Cell Type Ontology (CL) [14] and Cell Line Ontology (CLO) [30] respectively by using OntoFox, a web-based tool for retrieving and extracting ontological terms and axioms [31].

Given the above three types of host cells and two types of Brucella strains, we have asserted several subtypes of host-Brucella interactions using the format of 'host cell Brucella interaction'. Specifically, six subtypes of hostBrucella interactions were asserted:

1) macrophage - smooth Brucella interaction

2) macrophage - rough Brucella interaction

3) dendritic cell - smooth Brucella interaction

4) dendritic cell - rough Brucella interaction

5) epithelial cell - smooth Brucella interaction

6) epithelial cell - rough Brucella interaction.

Different agents participate in each of the host-Brucella interactions. For example, the agents in macrophage-Brucella interactions include:

1) 'Brucella' agent in 'macrophage - Brucella interaction'

2) 'Brucella' agent in 'process of establishing Brucella infection in macrophage'

3) ('macrophage' or 'macrophage cell line cell') agent in 'macrophage - Brucella interaction'

4) ('macrophage' or 'macrophage cell line cell') agent in 'macrophage anti-Brucella process'
5) 'macrophage-Brucella interaction' has_part 'process of establishing Brucella infection in macrophage'

6) 'macrophage-Brucella interaction' has_part 'macrophage anti-Brucella process'

Figure 1 illustrates the above triples and gives six subtypes of macrophage-Brucella interactions.

It is noted that the above categorizations do not count on the interactions between Brucella and host organ (e.g., spleen) or the whole host at an organism level. As Brucella is an intracellular bacterium, the macrophageBrucella interaction is critical to the outcome of the host-Brucella interaction [32]. In this article, we will primarily use the macrophage-Brucella interaction processes as an example for modeling the host-Brucella interactions.

\section{Ontology representation of Brucella invasion, trafficking, and replication inside host cells}

As an intracellular bacterium, the invasion, survival and replication of Brucella inside host cells are crucial to Brucella's lifecycle. The ultimate goal of Brucella is to propagate in their preferred niche in host cells (particularly the macrophages), where they can reach extensive replication and subsequently transmitted to new host cells. The intracellular life of Brucella is a subject of intensive scientific research [28, 32].

Specifically, through different modes of entry into a macrophage (details given in the following section), a smooth or rough Brucella cell will enter a Brucella-containing vacuole $(\mathrm{BCV})$ inside a macrophage. The $\mathrm{BCVs}$ containing smooth and rough Brucella cells undergo different intracellular trafficking pathways. Smooth BCVs become mature replicative niche, where the bacteria undergo extensive intracellular replications. In such a replicative niche, programmed macrophage cell death is prevented, which is beneficial for the intracellular Brucella. In contrast, rough bruellae are fused with lysosome and cannot

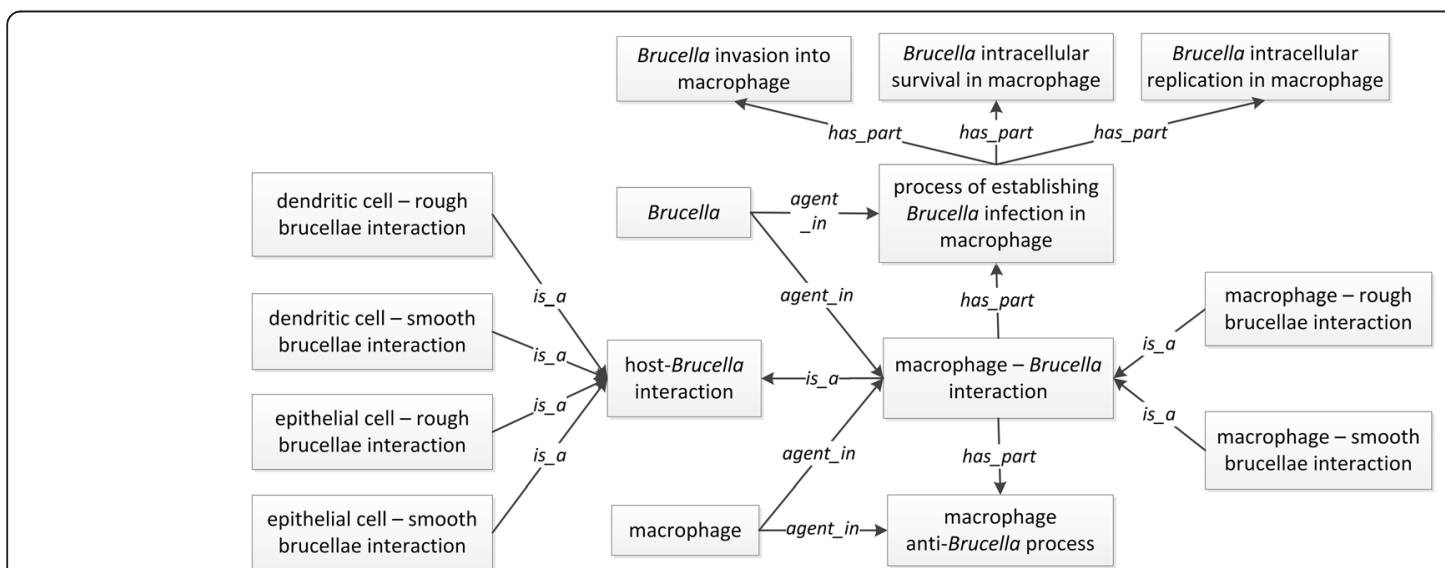

Fig. 1 Ontological representation of various interactions between Brucella and host cells 
replicate inside macrophages [28]. The rough $\mathrm{BCV}$ will undergo programmed cell death [25, 33-35]. The process of rough Brucella interacting with macrophages precedes the process of programmed macrophage cell death, which is beneficial for the host.

\section{Brucella: from entry into host cell to replication niche}

Smooth and rough Brucella strains utilize different mechanisms of entry into host cells. The Brucella LPS O polysaccharide is a critical molecule for interaction with lipid rafts within the plasma membrane of a host cell. The lipid rafts mediate the internalization of Brucella by phagocytes and nonprofessional phagocytes in a manner that leads to the development of the replicative niche [28]. The ontological representation of Brucella entry into macrophages and other related processes is shown in Fig. 2.

As shown in Fig. 2a, a smooth Brucella LPS (part of Brucella) and a lipid raft from a macrophage (part of macrophage) are both agents in the process of the interaction between smooth LPS and macrophage lipid raft. A lipid raft is composed of cholesterol (CHEBI_16113), ganglioside GM1 (CHEBI_61048), and glycosylphosphatidylinositol (CHEBI_24410). The LPS-lipid raft interaction leads to the engulfment of smooth Brucella into a macrophage, preceded with the formation of an early phagosome containing smooth Brucella. This early phagosome does not fuse with late endosome or lysosome [28]. The smooth Brucella containing vacuole (BCV) is acidified and trafficked to an endoplasmic reticulum (ER), where the BCV is fused with ER membrane. The fusion event leads to the formation of ER-derived replication niche of smooth Brucella, where the intracellular replication takes place. It is noted that only a small percentage of all invading Brucella cells will survive and achieve their goal of intracellular replications through the trafficking pathway.

Compared to smooth Brucella, rough Brucella cells have a different fate (Fig. 2b). Since rough Brucella does not have LPS O polysaccharide, rough Brucella enters into a macrophage via direct macrophage engulfment rather than the lipid raft-dependent engulfment [36]. The early rough Brucella-containing phagosome is fused with lysosome to form a rough Brucella-containing phagolysosome, where the invading bacterium is killed by the macrophage [36].

To formalize the representation of Brucella intracellular trafficking pathways, five formal relations defined in BFO were used: participates in, initially participates in, transformation of, begins to exist during, and starts_during. The terms participates_in and its subclasses initially participates in and begins_to_exist_during are three relations between continuant and process. If a continuant $c$ begins to exist during a process $p$, it infers that continuant $c$ does not exist before $p$ starts. If a continuant $c$ initially participates in a process $p$, it infers that $p$ cannot start without the existence of continuant $c$. Transformation_of links continuants in a similar fashion as preceded_by linking processes. Specifically, if continuant

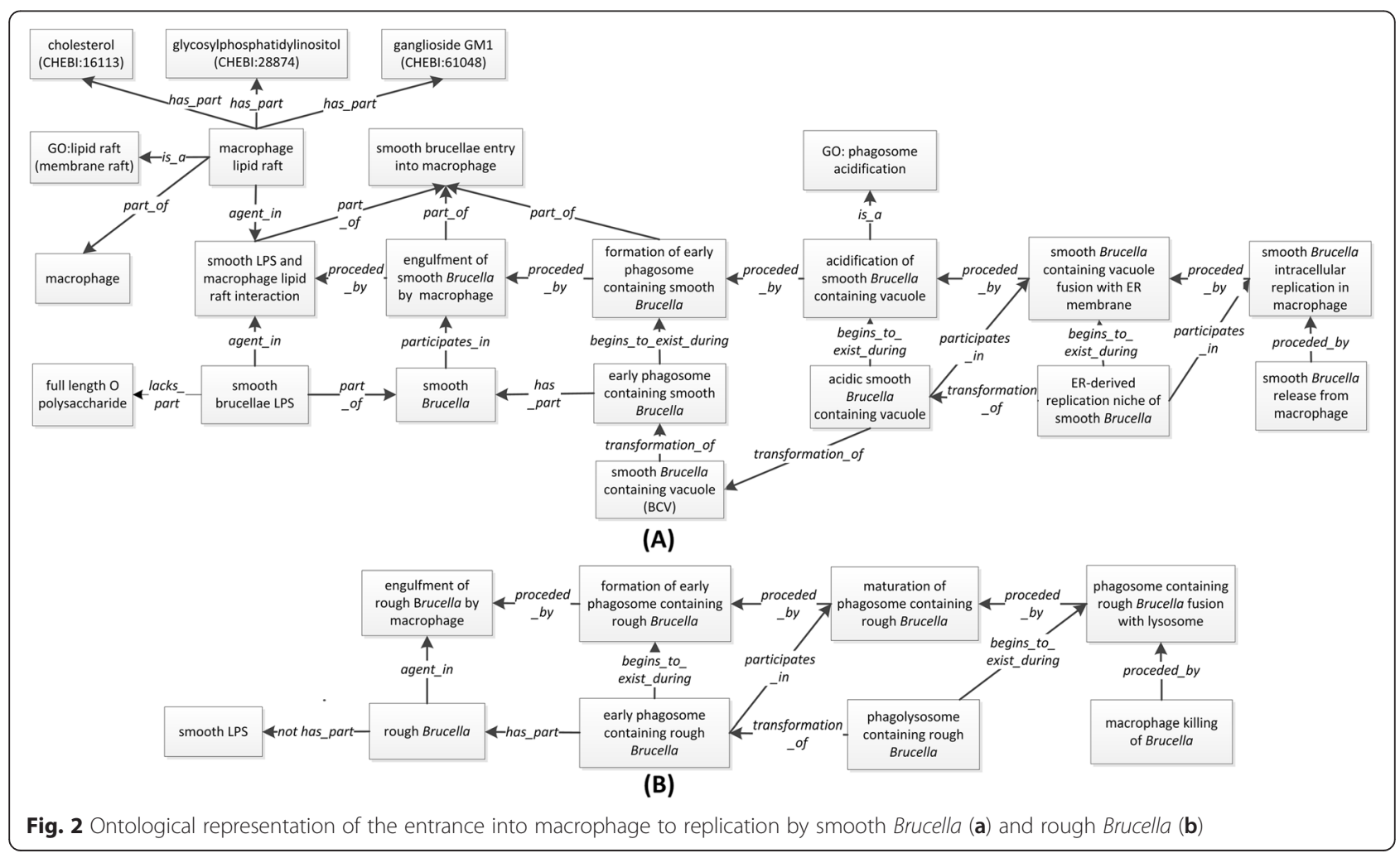


$c 1$ transformation_of continuant $c 2$, it infers that $c 2$ exists earlier than $c 1$. The term starts_during represents a relation between two processes. Taken these five relations together, the following IR2 is developed to infer the temporal relations between two processes:

(IR2) IF $c 1$ participates in $p 1, \cap c$ initiatively participates in $p 2, \cap c 2$ begins_to_exist_during $p 1$, $\cap c 2$ transformation_of $c 1$, THEN $p 2$ starts_during $p 1$

For example, based on the biological fact that an early smooth Brucella-containing phagosome is transformed to smooth Brucella-containing vacuole (Fig. 2), the following statements are represented in IDOBRU:

1. 'acidic phagosome containing smooth Brucella' participates in 'smooth Brucella containing phagosome fusing with ER membrane'

2. 'ER-derived replication niche of smooth Brucella' initiatively participates in 'smooth Brucella intracellular replication in macrophage'

3. 'ER-derived replication niche of smooth Brucella' begins_to_exist_during 'smooth Brucella containing phagosome fusing with ER membrane'

4. 'ER-derived replication niche of smooth Brucella' transformation_of 'acidic phagosome containing smooth Brucella'

Based on IR2, it is inferred that 'smooth Brucella intracellular replication in macrophage' starts_during 'smooth Brucella containing phagosome fusing with ER membrane'. The inferred statement is biologically valid [6].

As described in the Methods section, IR2 has been added to IDOBRU using the SWRL syntax (Additional file 1).

\section{Representing intracellular survival of smooth Brucella inside macrophages}

While a smooth Brucella-containing vacuole is trafficking within a host cell, the bacteria inside the vacuole encounter formidable environmental stresses such as the exposures to reactive oxygen (ROS) and nitrogen species (RNS), acidic $\mathrm{pH}$, nutritional deprivation, and lytic peptides contained in lysosomes [28]. To withstand these environmental stresses, Brucella has developed different strategies.

IDOBRU uses several 'smooth Brucella resistance' subclass terms to represent the resistant processes enabling smooth Brucella to survive under the stressful environments within macrophages. Examples of such terms are: 'smooth Brucella resistance to nutrient deprivation', 'smooth Brucella resistance to antimicrobial peptide', 'smooth Brucella resistance to nitrosative stress inside BCV', 'smooth Brucella resistance to oxidative stress inside BCV', and 'smooth Brucella resistance to acidity stress inside $\mathrm{BCV}$ '. The 'part of' relation was used to link above processes to 'process of establishing smooth $\mathrm{Bru}$ cella survival in macrophage' in the ontology.

The abilities of smooth Brucella resistance to those stressful conditions were represented as different types of 'disposition'. The IDO term 'protective resistance' is used as their direct mother term in virtue of protecting Brucella from different stresses. Smooth Brucella has the dispositions including 'nutrient deprivation resistance disposition', 'antimicrobial peptide resistance disposition', 'oxidative stress resistance disposition', 'nitrosative stress resistance disposition', and 'acidic stress resistance disposition'. These dispositions are realized in relevant resistance processes and inhere in a 'smooth Brucella strain'. For example, the 'nutrient deprivation resistance disposition' of smooth Brucella is realized in the process of 'smooth Brucella resistance to nutrient deprivation', and 'smooth Brucella strain' is the agent participating in the process (Fig. 3).

As the interactions are between host and pathogen, the response actions of macrophage cells are represented in IDOBRU using four biological process terms: 'nitricoxide synthase biosynthetic process' (GO_0051767), 'reactive oxygen species metabolic process' (GO_0072593), 'acidification of BCV in macrophage' (IDO_0100758), and 'macrophage antimicrobial peptide production' (IDO_ 0100759). All these processes are part of the 'macrophage anti-Brucella process'. The unfolds_in relation is used to capture the fact that those processes take place inside a macrophage cell (Fig. 3).

\section{Representing Brucella pathogenesis mechanisms}

In macrophages, the majority of $\mathrm{BCV}$ s fuse with lysosomes and the bacteria are killed and degraded in the early hours of internalization [36]. Approximately $10-30 \%$ of internalized Brucella cells are able to survive and undergo the intracellular trafficking of the host cell [37]. The molecular mechanisms of Brucella pathogenesis are responsible for all kinds of interactions with their mammalian hosts. Virulent Brucella employs several strategies and uses many virulence factors to establish and maintain persistent intracellular residence in host cells. Intracellular Brucella also alters biological functions of professional phagocytes, resulting in the losing of their robust antigen-processing capacity. In order to prevent more hostile extracellular environment, virulence Brucella is able to prevent the programmed cell death of infected macrophages [38]. Brucella pathogenesis relies on the presence of many Brucella virulence factors and their interactions with the host defense system. Two examples are generated here to illustrate how IDOBRU represents the interactions between host and bacterial genes, proteins and pathways.

\section{Representing type IV secretion system}

Bacterial type IV secretion systems (T4SS) are often critical to selective translocation of proteins and DNA- 


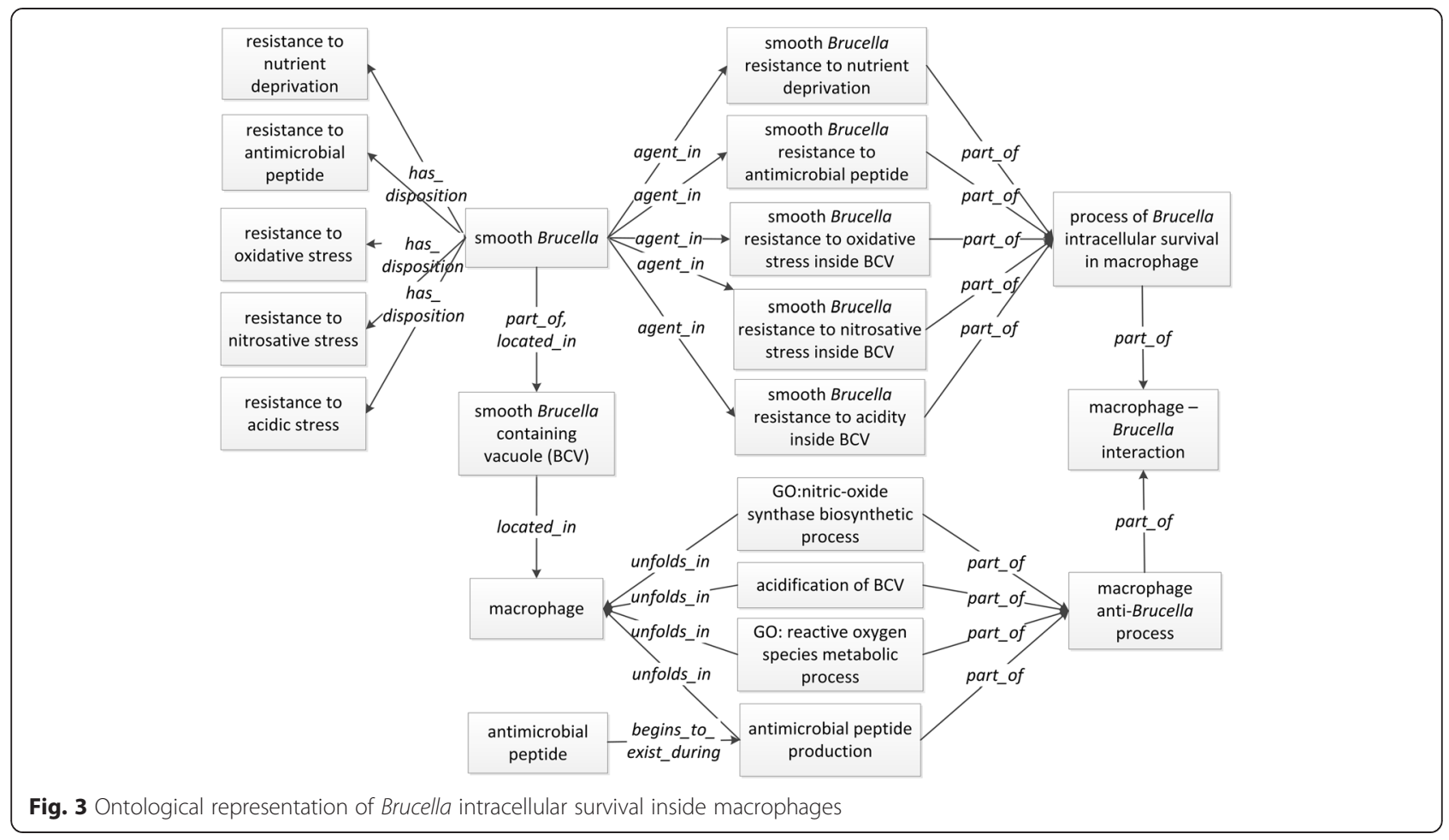

protein complexes [39]. Brucella T4SS, encoded by the $\operatorname{vir} B$ operon, is a major virulence factor system [38-41]. Brucella T4SS virB operon includes 12 genes whose expression is specifically induced by the phagosome acidification after Brucella phagocytosis [40]. Brucella T4SS is required for smooth Brucella trafficking to replication niches and intracellular survival inside host cells. In rough strain of Brucella, T4SS expression is important for Brucella cytotoxicity in macrophages [38].

Figure 4a represents Brucella T4SS and its roles in the pathogenesis of smooth Brucella. The 'Brucella virB operon expression' is preceded by 'acidification of smooth Brucella containing phagosome'. The Brucella VirB proteins, encoded by the Brucella virB operon, start to exist with the expression of the virB operon genes (begin to_exist_during). The process of binding a virB promoter regulates the T4SS virB operon expressions. For example, $\mathrm{VjbR}$, a LuxR-type quorum-sensing regulator, binds on the virB promoter, and activates Brucella virB operon expression [42]. Therefore, VjbR regulates Brucella T4SS directly and subsequently has an impact on the effectors of T4SS protein secretions. As shown in Fig. 4a, VjbR is an agent in the Brucella virB promoter binding process, which leads to positive regulation of the Brucella virB operon expression.

Brucella T4SS acts as a translocator of Brucella proteins or DNA into a macrophage [42]. Entities translocated or secreted by Brucella T4SS are termed as 'Brucella T4SS effectors', which regulate different processes essential to
Brucella intracellular trafficking or replication. A Brucella T4SS effector is defined as "a molecular entity that bears the Brucella T4SS effector role" [40]. The 'Brucella T4SS effector role' is a new IDOBRU term that is defined as: "a role that inheres in a Brucella protein or a DNA upon which the Brucella T4SS acts, and as a result, the protein or DNA is secreted out of the bacterium."

As an example of a Brucella T4SS effector, Brucella protein RicA (Rab2 interacting conserved protein A), is represented in IDOBRU to illustrate the Brucella T4SS virulence mechanism (Fig. 4). Brucella RicA, encoded by a Brucella gene BMEI0736, binds to human small GTPase protein Rab2 [43]. RicA is translocated from B. abortus to infected macrophages. However, this phenomenon does not occur when a Brucella virB mutant infects the macrophage cell. Rab2 also coordinates the retrograde Golgi-to-ER transport [44]. The Rab2 is essential for the formation of the fusion between BCV and ER, which results in the Brucella replication niche [45]. As shown in Fig. 4b, Brucella T4SS participates in the process of translocation of RicA into a macrophage' as an agent. Brucella protein RicA bears 'Brucella T4SS effector role', and it participates in the processes of 'translocation of RicA into macrophage' and 'RicARab2 binding'. The process of 'RicA binding to Rab2' leads to the formation of 'RicA-Rab2 complex'. Rab2 participates in the regulation of Golgi-to-ER transportation, which regulates the 'retrograde Golgi-to-ER transport'. Overall, Fig. 4b provides a detailed explanation to an axiom stated in Fig. 4a: 'Brucella T4SS effector 


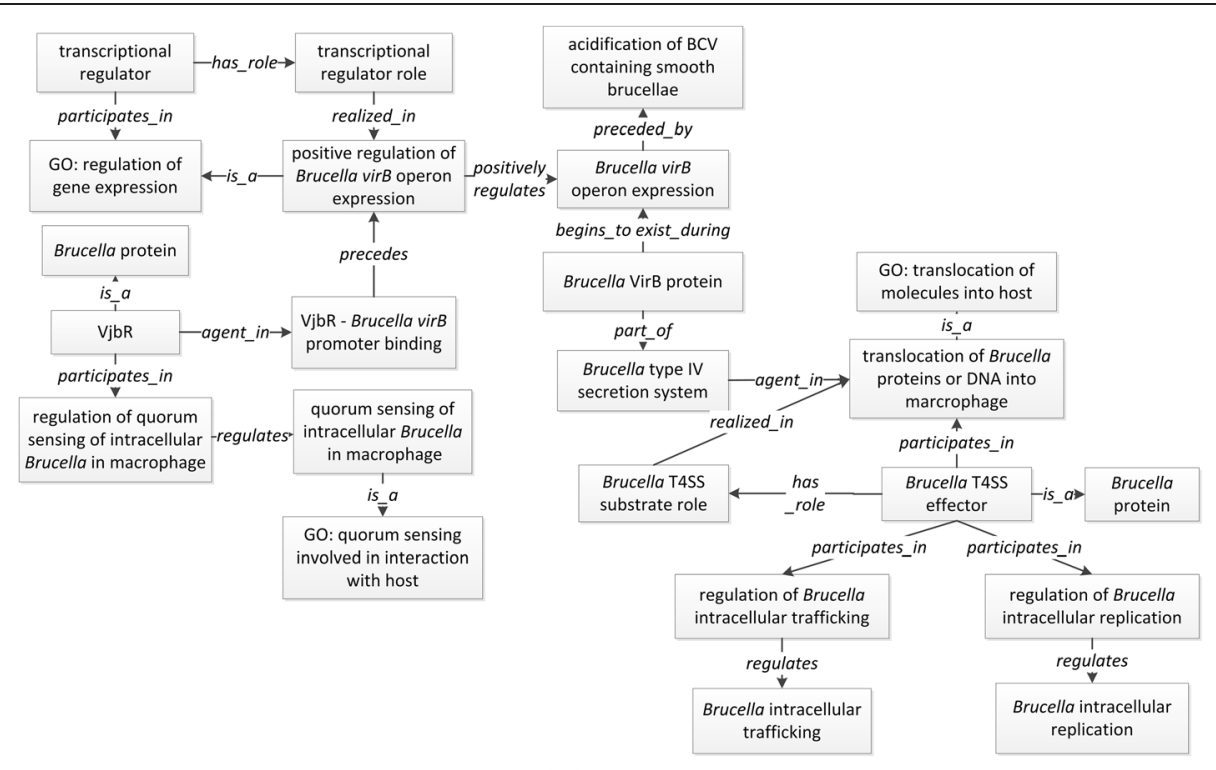

(A)

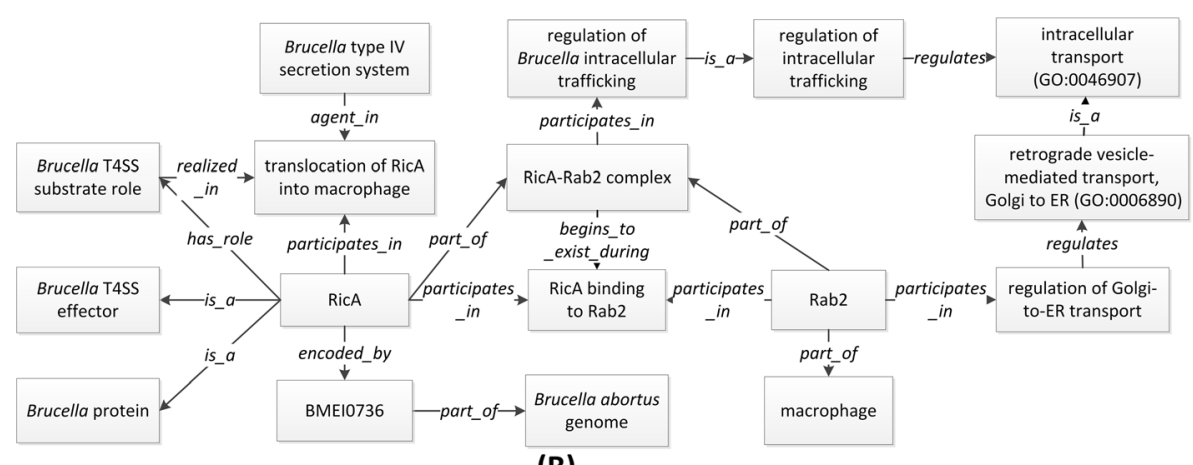

(B)

Fig. 4 Ontological modeling of Brucella type IV secretion system and its effects. a The general Brucella type IV secretion in Brucella (a) and example of RicA as T4SS effector (b)

participates in the regulation of Brucella intracellular trafficking'.

\section{Representing Brucella erythritol metabolism}

As described earlier, one of the intracellular environmental stresses that Brucella faces is nutritional deprivation. Brucella uses alternative metabolism pathways to obtain carbon, nitrogen, oxygen, phosphorus, sulfur and metals from its intracellular host [5]. For example, an alternative pathway for Brucella to acquire carbon is through the erythritol metabolism $[28,46]$. The genome of attenuated B. abortus vaccine strain S19 includes a 703 nucleotide deletion on its ery operon. The deletion affects the gene eryC coding for an enzyme erythrulose-1-phosphate dehydrogenase (EryC) and another gene eryD that encodes for EryD, a regulator of ery operon expression [47]. The deletion is a cause of the attenuation characteristic of strain 19 [48]. The eryC mutant of $B$. suis also reduces its intracellular replication in macrophage cells [46].
To ontologically represent the virulent characteristic of EryC, we started by representing the whole process and its participants at the molecular level and organism level (Fig. 5):

1) The erythritol metabolism pathway is important for Brucella intracellular replication. The intracellular Brucella in a macrophage has the disposition of uptaking erythritol as the carbon source, which is realized in the relevant 'uptaking erythritol process'. The process of 'uptaking erythritol as carbon source' has 'Brucella erythritol catabolic process' as its part. The 'enzyme substrate role' of erythritol is realized in the erythritol catabolic process. The term 'enzyme substrate role' is defined as "A role that inheres in a protein or a compound upon which enzyme catalyzes. It is realized in the enzymatic reaction processes, where the molecules at the beginning of the process, called substrates, are converted into 


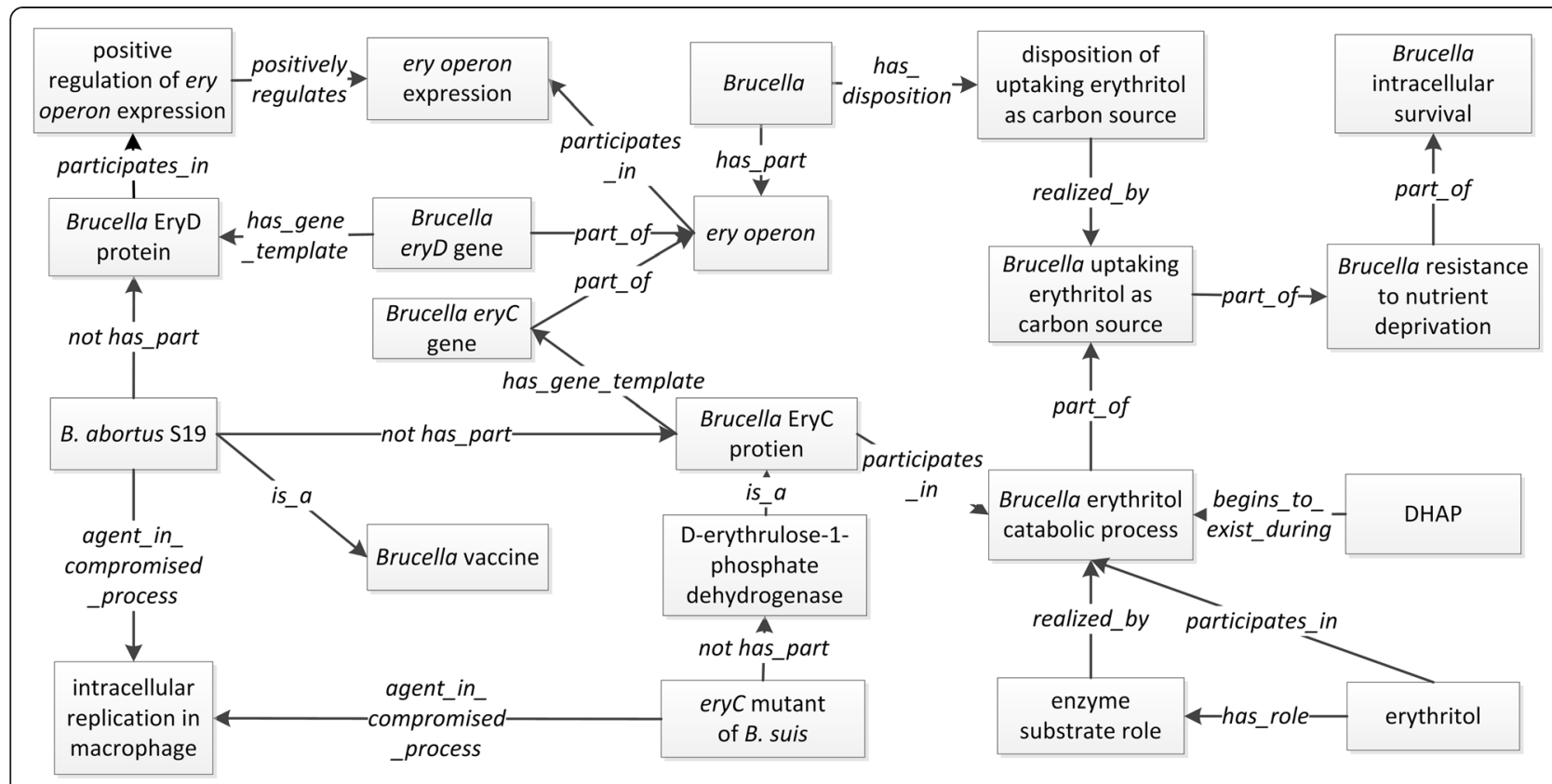

Fig. 5 Ontological representation of Brucella erythiritol metabolism and its involvement in Brucella pathogenesis

different molecules, namely products." The final product of 'erythritol catabolic process' is the dihydroxyacetone phosphate (DHAP), which is represented using the relation begins_to_exist_during as: 'DHAP begins_to_exist_during erythritol catabolic process'. The process of 'uptaking erythritol as carbon source' is a partial process of 'Brucella resistance to nutrient deprivation', which accordingly precedes and is required for the process of 'Brucella intracellular replication'. The relation preceded_by is used to denote the relation between these two processes. Both 'Brucella response to nutrient deprivation' and 'Brucella intracellular replication' are parts of the 'host-Brucella interaction' process (Fig. 5). It is noted that we did not model the detailed chemical reactions that are components of the whole erythritol catabolic process, because it is out of the scope of current IDOBRU development.

2) Brucella genes eryC and eryD involve in the erythritol metabolism pathway.

Both eryC and eryD are parts of the Brucella ery operon. The eryC gene encodes erythrulose-1phosphate-dehydrogenase enzyme (EryC), and it participates in the erythritol catabolic process. The ery $D$ gene encodes for an EryD protein that regulates the ery operon expression [47]. The object property regulates is a relation between processes, and it is used in GO to represent the fact that a process $A$ has a direct influence on another process $B$ such that it controls some aspects of how process B unfolds [49].
Therefore, the protein EryD regulates the ery operon expression (Fig. 5).

3) B. abortus vaccine strain S19 has a 703-nucleotide deletion which interrupts both the coding regions of $\operatorname{eryC}$ (BAB2_0370) and eryD (BAB2_0369) [48]. The deletion affects the $C$ terminal part of the Brucella protein EryC and the N-terminal part of the Brucella protein EryD [48]. Therefore, S19 lacks the intact EryD protein and EryC protein as its parts. In the other example, the eryC mutant of B. suis has no EryC protein as its part, and it is an agent in the process of 'reduced intracellular replication in macrophage' [46]. 'Reduced intracellular replication in macrophage' is a compromised intracellular replication process in macrophage.

\section{Representing host immune responses to Brucella infection} Virulent Brucella is a stealthy bacterium that hijacks many host immune mechanisms to serve its own survival and replication inside a host [6]. As introduced above in the Brucella pathogenesis section, virulent Brucella is able to replicate inside macrophages which are typically powerful innate immune cells. Brucella can survive in replicative phagosomes inside macrophages where nutrients are difficult to obtain. The Brucella-containing phagosome does not fuse with bactericidal lysosomes [6]. Furthermore, to maintain the bacterial natural living niche, virulent Brucella prevents the programmed macrophage cell death. However, live attenuated Brucella strains, including Brucella cattle vaccine RB51 [50], induce apoptosis or other 
types of programmed cell death of infected macrophages, which destroys the Brucella living niche and exposes the bacteria to the most hostile extracellular environment $[25,34]$. Therefore, the programmed cell death process benefits the host. Below we ontologically represent and analyze the process using the example of live attenuated rough $B$. abortus strain RB51, which is a cattle brucellosis vaccine licensed and used in the USA and many other countries [50].

Our previous wet-lab studies have shown that RB51 and many other rough attenuated Brucella strains induce caspase-2-mediated pro-inflammatory cell death in macrophages through the release of cytochrome $c$ from mitochondria $[34,35]$. RB51 has an insertion within $w b o A$ gene that leads to the deficiency of Brucella LPS O polysaccharide and results in its rough phenotype [51]. Figure 6 illustrates ontological representation of RB51-induced capspase-2-mediated macrophage cell death. In this representation, RB51 has no intact $w b o A$ gene that encodes for an enzyme involved in the biosynthesis of Brucella abortus O-polysaccharide [52]; therefore, RB51 is lack of Brucella abortus $\mathrm{O}$-polysaccharide. RB51 is an agent in the process of its infecting a macrophage, which is a part of the 'macrophage-RB51 interaction' process. The 'RB51 infection of macrophage' triggers (RO:precedes) three processes: 'activation of caspases-2', 'positive regulation of macrophage programmed cell death', and 'positive regulation of macrophage necrotic cell death'. The 'activation of caspases-2' leads to (RO:precedes) 'positive regulation of macrophage programmed cell death'. The 'positive regulation of macrophage cell programmed death' positively regulates the 'apoptotic macrophage cell programmed death', which leads to 'necrotic macrophage cell death'. The 'necrotic macrophage cell death' is positively regulated by the 'positively regulation of macrophage necrotic cell death'.

From a macrophage's perspective, the macrophage has two opposite dispositions: 1) the 'disposition of undergoing programmed cell death' that is realized by attenuated RB51 infection of macrophage; and 2) the 'resistance to programmed cell death' that is realized by 'virulent Brucella infection of macrophage'.

\section{Ontological representation and queries of virulence factors and associated host-Brucella interactions Ontology classification of Brucella host-Brucella interactions involving virulence factors}

We previously defined a 'Brucella virulence factor' as "virulence factor that bears Brucella virulence factor disposition" [10]. The 'Brucella virulence factor disposition' is defined as "a disposition borne by a biological macromolecule produced by Brucella spp. that is the disposition to improve survival of the pathogen in a host, improve transmission of the pathogen to a host, or cause pathological processes in a host". To further expand the definition, virulence factors are ontologically classified in this article to be critical to five pathogen virulence (or microbial pathogenesis) processes:

1) colonization of a niche in the host (this includes adhesion to cells);

2) evasion of the host's immune response;

3) inhibition of the host's immune response;

4) entry into and exit out of cells (if the pathogen is an intracellular one);

5) absorption of nutrition from the host.

As shown in the modeling of T4SS and eryC described above, a Brucella virulence factor is involved in at least one critical process as part of the host-Brucella interaction, or a process precedes the critical process. Although many Brucella molecules participate in the host-Brucella interaction processes, not all of them contribute to the virulence of the Brucella. One method to confirm the status of a molecule being a virulence factor is knock-out experimental evidence, where the pathogen without this molecule realizes a "reduced" or "abolished" virulence disposition during the host-Brucella interaction.

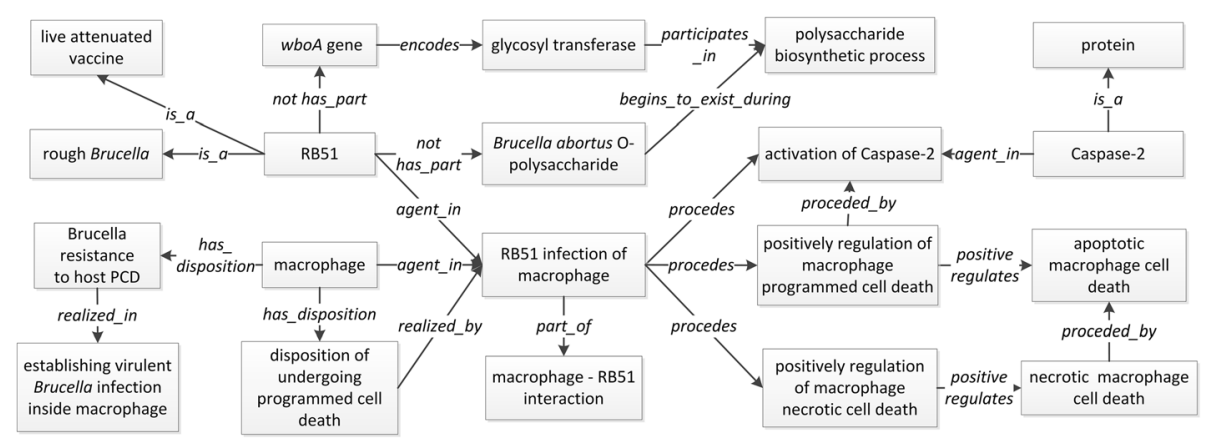

Fig. 6 Ontological representation of Brucella vaccine strain RB51-induced macrophage cell death 


\section{Ontology representations of Brucella virulence gene mutants}

The major category of virulence factors are protein virulence factors that are encoded by virulence genes. Compared to the original IDOBRU that includes 245 Brucella virulence factors [10], current IDOBRU has been expanded to include 432 experimentally-verified Brucella virulence factors. All these virulence factors are experimentally verified. The gene mutation followed by experimental examination of the virulence of the gene mutant inside a host (i.e., the host organism or host cell) is the major method to detect the status of a pathogen protein as a virulence factor.

Figure 7 shows how IDOBRU represents a virulence gene mutant that lacks an intact protein virulence factor. Specifically, a gene mutant is represented in IDOBRU as a mutant that does not has_part a gene, which also results in the lack of an intact protein (Fig. 7a). The original IDOBRU used IDO ontology identifiers with the "IDO_" to represent Brucella genes and proteins. However, Brucella genes and proteins may be used in other ontologies such as the Vaccine Ontology (VO) [53, 54]. The usage of IDO-specific identifiers does not support data integration and resource interoperability. As detailed in the Methods section and shown in Fig. 7, in the new version of IDOBRU, we have imported the Ontology of Genes and Genomes (OGG) IDs (Fig. 7b) and Protein Ontology (PRO) IDs (Fig. 7c) to represent the genes and proteins of Brucella virulence factors. OGG is a relatively new ontology that represents specific genes in different species [19]. PRO is an ontology of protein entities [20]. To link the gene and protein entities, we have adopted the PRO relation 'has_gene_template' to represent a protein encoded by a gene (Fig. 7c), and the relation 'encodes' to represent a gene encoding a protein.

\section{Description rules to define virulence factors}

To establish logical reasoning for a virulence factor, we developed five description rules as defined below. In the formulation of these rules, $o$ denotes an organism $\mathrm{O}, g$ and $g^{\prime}$ denote genetic materials, $e$ denotes a molecular entity, $p$ denotes a process, $i$ denotes a host-pathogen interaction process, and mo denotes a mutant of $\mathrm{O}$.

\section{(IR3) IF $o$ has_part $g, \cap g$ encodes $e$, THEN $o$ has_part $e$}

IR3 means that if an organism has part of a gene that encodes for a molecular entity (i.e., gene product such as protein), then this organism has part of the molecular entity.

(IR4) IF mo has_part $g^{\prime}, \cap g^{\prime}$ derives_from $g, \cap\left(g^{\prime}\right.$ not has_part part of $g$ ) $\cap$ genome of $o$ has_part $g$, THEN genome of mo not has_part $g$

IR4 means that if a mutant of an organism has an artificially altered gene $g$ ' that is derived from $g$, either by an insertion or partial deletion, then the genome of the mutant has no intact $g$ as its part. When the $g$ is fully deleted (i.e., $g$ gene knock-out) from mutant mo, the above rule will not apply. In this case, we simply assert that genome of mo not has_part $g$ (see below).

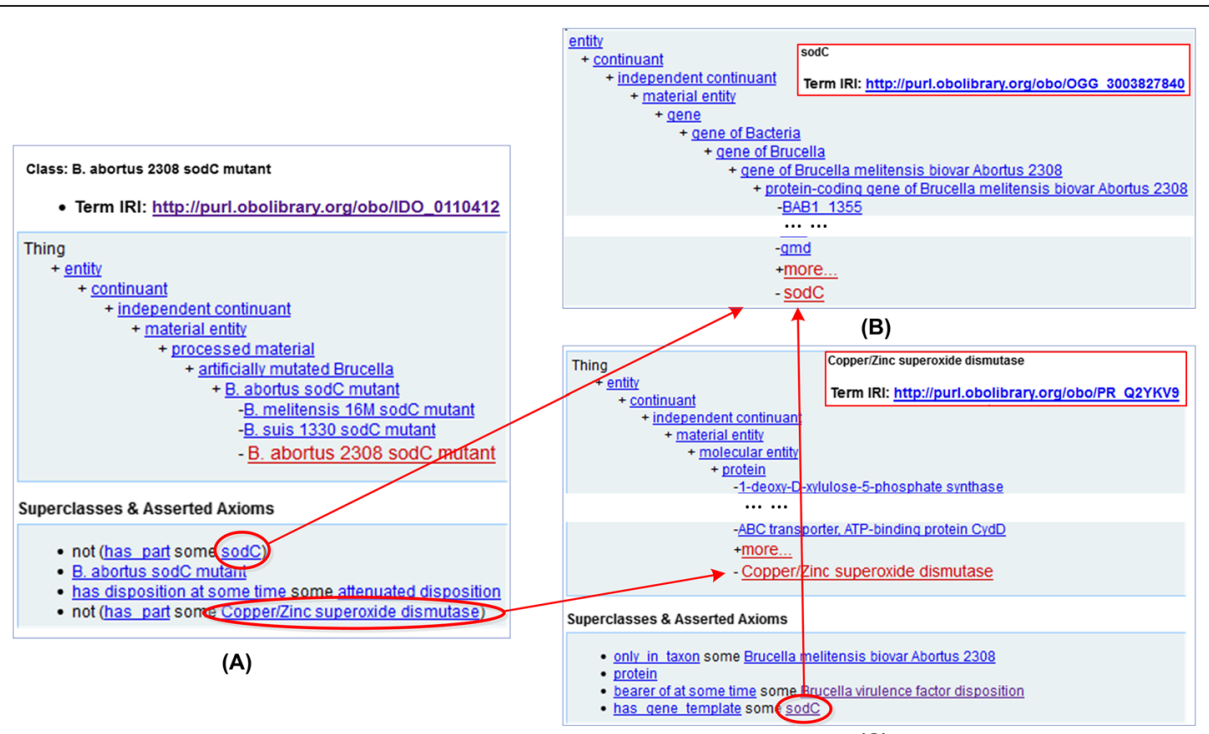

(C)

Fig. 7 IDOBRU representation of a Brucella virulence gene mutant. The example shown here is B. abortus strain 2308 sodC mutant (a). This mutant has a mutation of strain $2038 \operatorname{sod} C$ gene $(\mathbf{b})$, which encodes a protein called Copper/Zinc Superoxide Dismutase (SOD) (c). The relation 'has_gene_template' is used to link the protein to the gene. The screenshots came from the IDOBRU page in Ontobee [61] 
(IR5) IF genome of mo not has_part $g$, THEN mo not has_part $g$

If the genome of mutant has no intact gene $g$ as its part, the mutant has no $g$ as its part.

(IR6) IF genome of mo not has_part $g, \cap g$ encodes $e$, THEN mo not has_part $e$

IR6 means that if the mutant has no intact gene $g$ as its part, and the gene $g$ encodes the molecular entity $e$ in the non-mutated organism, then the mutant has no intact $e$ as its part.

IR7 was given as a final inference rule for inferring a virulence factor:

\section{(IR7) IF (mo has disposition at some time} attenuated disposition $\cap$ attenuated disposition realized in $i) \cap m o$ not has_part $e, \cap(m o$ agent_in_compromised_process $p \cap p$ is_a pathogen virulence process), THEN $e$ is_a virulence factor.

For example, as shown in Fig. 5, (B. abortus ery $C$ mutant not has_part EryC) AND (B. abortus eryC mutant agent_in_compromised_process Brucella intracellular replication in macrophage) AND (Brucella intracellular replication in macrophage is_a pathogen virulence process) means that EryC is_a Brucella virulence factor. According to IR6, if a gene $g$ encoding a protein $e$ is mutated from a mutant, the mutant does not have the intact protein $e$ any more. Since $e r y C$ is mutated from the ery $C$ mutant, we can infer that the eryC mutant does not have EryC.

We can therefore identify the biological process important to the pathogen virulence during the host-Brucella interaction. Using the same Fig. 5 example, (EryC participate_in Brucella erythritol catabolic process) AND (EryC mutant of $B$. abortus agent_in_compromised_process intracellular replication in macrophage), which means that the Brucella erythritol catabolic process is crucial to the intracellular replication in macrophage.

With the support of the above representations defined by IR3-7, we have annotated 269 virulence factors associated with various macrophage-Brucella interactions. IR7 is included in IDOBRU (Additional file 1: Figure S1).

\section{SPARQL queries of IDOBRU for virulence factors critical for host-Brucella interactions}

In this study, several SPARQL scripts were generated to query the information related to host-Brucella interactions. The details are provided below:

First, a simple SPARQL script was generated to query the number of protein virulence factors collected in IDOBRU (Additional file 2). Each virulence factor protein is 'bearer of at some time' (BFO_0000053) some 'Brucella virulence factor disposition' (IDO_0100116). The query identified 432 protein virulence factors.

The second SPARQL query identifies the processes in which Brucella virulence factors participate (Fig. 8). Specifically, the script queries what compromised processes Brucella virulence factor mutants get involved in. The relation 'agent_in_compromised_process' as described earlier is used here. In total, 11 biological processes, for example, 'Brucella entry into macrophage' (IDO_0100610) and 'Brucella intracellular trafficking' (IDO_0100983), were identified (Fig. 8). These processes are critical to Brucella pathogenesis inside host cells.

Third, those Brucella mutants that are attenuated inside macrophages during various macrophage-Brucella interactions were identified using SPARQL (Additional file 3). Each of these mutants is associated with a particular gene (represented in OGG) and a corresponding protein (represented in PRO) (Fig. 7). Therefore, the queries also provide us a way to extract those virulence genes and virulence factors important for macrophageBrucella interactions. In total, 269 gene mutants that are associated with 269 genes and protein virulence factors were found. The list of all these gene mutants is also provided in Additional file 3.

Fourth, the Brucella protein virulence factors important for Brucella intracellular replication inside macrophages were detected using two SPARQL queries (Additional file 4). A query identified 81 such virulence factors, and the other query provided the detailed list of these factors (Additional file 4).

It is noted that the inference rules IR3-7 provide the logic clues on the second and third sets of queries. Specifically, to search Brucella virulence factors important for intracellular replication in macrophages, first we retrieved all possible gene mutants. Each protein encoded by a gene mutated in a mutant is an agent involved in the compromised process of 'macrophage-Brucella interaction' (IDO_0100832) (Additional file 3: Figure S3) or 'Brucella intracellular replication in macrophage' (IDO_0100612) (Additional file 4: Figure S4). The relation 'agent_in_compromised_process' as described earlier is used here. Due to the mutation event, the intact Brucella protein (virulence factor) does not exist in the mutant organism (IR4 and IR6). Therefore, we are able to retrieve all the corresponding virulence factor proteins using the not has_part relation (Additional file 4: Figure S4).

\section{Discussion}

Compared to the original IDOBRU paper published in 2011 [10], current article included several novel contributions. First, while the original paper only includes one section with one figure in the topic of the host-Brucella interaction (i.e., virulence factor and pathogenesis), 


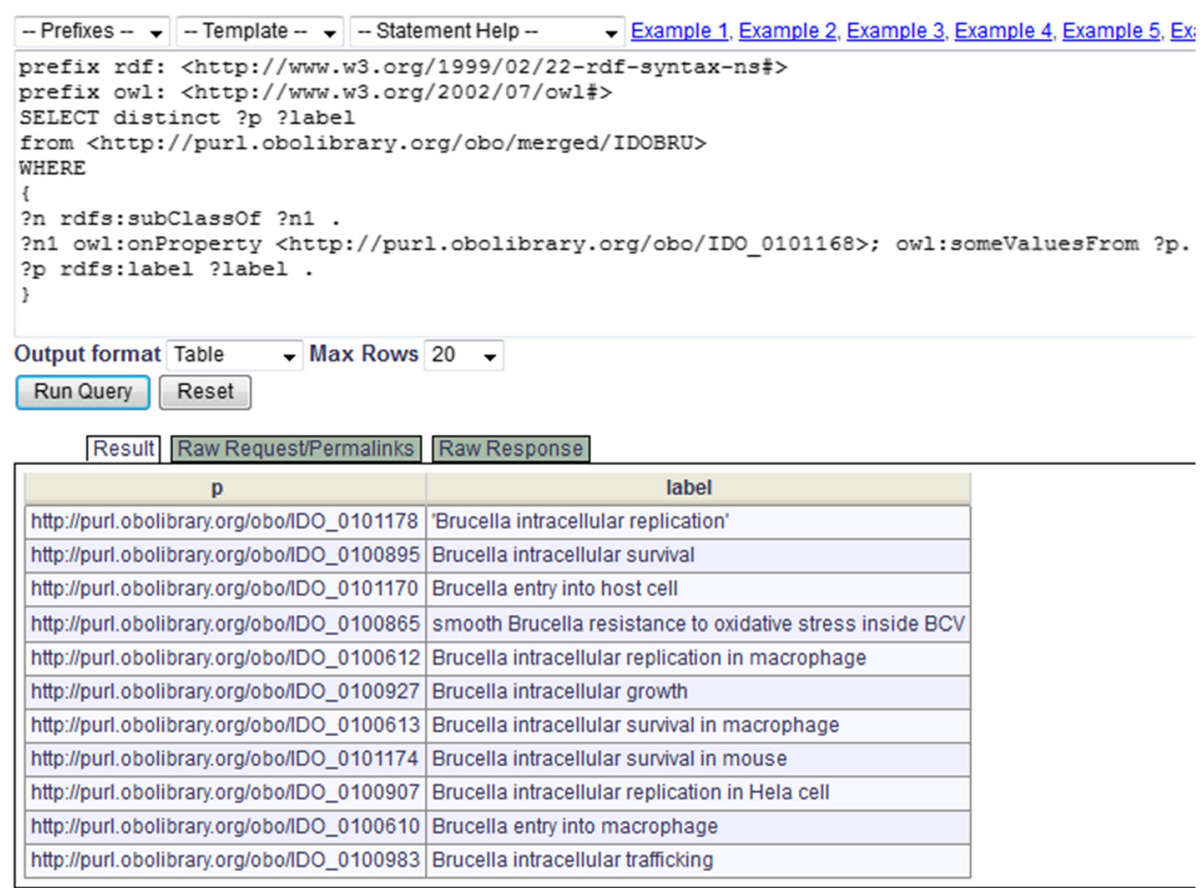

Fig. 8 SPARQL query of biological processes involving Brucella virulence factors. This script queries the compromised Brucella processes in which Brucella virulence gene mutants participate. The query was performed using the Ontobee SPARQL web-interface (http://www.ontobee.org/sparql). The IDO_0101168 in the script is 'agent_in_compromised_process'

current article focuses on the modeling and analysis of different aspects of the host-Brucella interactions. Specifically, this article first ontologically differentiates smooth and rough Brucella phenotypes and how each phenotype is related to Brucella virulence. Six host-Brucella interaction subtypes are categorized, and the agents participating in any of the subtypes (i.e., macrophage-Brucella interaction) are defined. IDOBRU is also used to represent detailed processes of Brucella invasion, trafficking, and replication inside host cells. Examples described in this article include two major Brucella pathogenesis mechanisms: the Type IV secretion system (T4SS) and the erythritol metabolism. In terms of host immune response against Brucella infection, the inhibition or promotion of host programmed cell death is specifically modeled using IDOBRU. Regarding the virulence factors, this article for the first time ontologically classifies five different types of host-pathogen interaction processes where virulence factors may play a critical role. Approximately 200 more virulence factors have been included in current IDOBRU since the original IDROBRU publication. Second, seven specific inference rules are generated and described in this paper for reasoning related to host-Brucella interactions and Brucella virulence factors. The layout and implementation of these inference rules provide more power in using IDOBRU for computerassisted reasoning. Third, this article introduces an updated style of representing Brucella genes and proteins. Instead of using IDO IDs to represent genes and proteins, the updated IDOBRU imports OGG and PRO IDs for more authentic representations of Brucella genes and proteins. Such gene/protein representations support ontology reuse and interoperability. Fourth, this article provides many SPARQL scripts that demonstrate the applications of IDOBRU. Furthermore, many more ontology terms have been added to IDOBRU. Compared to the original IDOBRU version published in 2011 that includes 1503 terms, current version 1.2.79 includes 3488 terms. We have more than doubled the numbers of the terms in the IDOBRU ontology, clearly showing our progress in the IDOBRU development.

Other literature reports exist for ontological modelling of host-pathogen interactions. The Plant-Associated Microbe Gene Ontology (PAMGO) Consortium uses GO for modeling host-pathogen interactions based on the investigation of plant-associated symbionts [55]. Their efforts yielded a group of GO biological process terms that capture the processes occurring between hosts and symbionts (from mutualists to pathogens). PAMGO is focused on representing processes. Representation of host participants (e.g., organelle like ER, and cell membrane) and pathogen details (e.g., LPS of Brucella, Brucella proteins) was not covered. IDO-core provides many top level terms in the area of host-pathogen interactions, such as establishment of localization in host (IDO_0000625). As an extension ontology of IDO-core, the Malaria Ontology team developed several terms related to malaria-host interact 
processes, such as 'inhibition of invasion' and 'responsiveness to host cue' [56]. Another ontology named Host Pathogen Interaction Ontology (HPIO) found on NCBO BioPortal is current under development, which aims to describe the host-pathogen interactions between Salmonella bacteria and swine, also as an extension of IDO-core. However, HPIO does not represent the interactions between host and pathogens extensively. None of the above efforts covers the participants of the interaction processes and relations between those processes. In contrast, IDOBRU represents various participants in the interaction processes between Brucella and its hosts with details at the organism, cell, and molecular levels. IDOBRU is used as a framework to model different proteins, complexes and interaction processes such as how virulence factors play a role in the Brucella-host interactions and how the interactions happened as a series of sequential events. To support data standardization and exchange, formal relations are applied in the IDOBRU modeling. To the best of our knowledge, our IDOBRU modeling and representation of various areas of host-Brucella interactions represent the first comprehensive host-pathogen interaction ontological analysis.

The host-Brucella interaction is modeled in IDOBRU as a process with many specific interaction subclasses. The interactions between Brucella and hosts are Brucella-specific and host (e.g., host organism or cell) - specific. For example, the pathway of Brucella entry into macrophage differs from the bacterial entry into epithelial cell, and the immune response and intracellular trafficking induced by Brucella infection differ among professional and nonprofessional phagocytes [57]. Therefore, it is important to classify different subtypes of host-Brucella interactions by host cells. The macrophages are emphasized in this study since macrophage is likely the most critical host cell type in terms of host-Brucella interactions [32]. More ontological representations with other host cells are needed. Similarly, we will need to classify the subtypes based on host organism or host organs as well, since the infection of any Brucella strain has its host preference. These aspects are critical to the development of host-specific Brucella vaccines.

Compared to well-studied model organisms such as $E$. coli, Brucella is less studied, and the coverage of Brucella research is often unbalanced and limited. For example, middle products and enzymes involved in the erythritol catabolism pathway in Brucella are not available in PRO or ChEBI. We communicated the PRO and ChEBI groups and submitted related middle product terms to ChEBI and enzyme terms to PR. For example, over 1000 Brucella strain specific proteins were submitted to PR. Although the enzyme databases and knowledge bases are well developed and contain authentic comprehensive information, the erythritol catabolic pathway is not included in well- known enzyme databases such as BRENDA (http:// www.brenda-enzymes.org/). GO has very few gene products from Brucella annotated with experimental evidence codes (http://www.geneontology.org/GO.evidence.shtml).

The inference rules (IR3-IR7) generated in this study provide a framework on inferring virulence factors. These rules can be used for validating the ontology and populating the ontology. These inference rules can also be generalized to other pathogen. The inference rules represent knowledge and provide a format for computer-understandable automated reasoning. These rules offer explicit and transparent assumptions for representing virulence factors in the case of the host-Brucella interaction. Based on the inference rules and available data, a computer will be able to assess if a material entity is a specific virulence factor. Since the mechanisms of virulence are different from species to species, more inference rules may be developed with different types of pathogens (e.g., HIV virus).

SPARQL provides a powerful method for querying and analyzing the data in an ontology [58]. For example, our queries identified 269 protein virulence factors related to macrophage-Brucella interactions; and among these proteins, 81 are important for intracellular replication within macrophage from the knowledge stored in current IDOBRU. Note that virB1, virB5, virB8, virB9 and $\operatorname{virB10}$ are in the above list, which validates the modeling of T4SS mechanism in this paper. In addition, we have identified 11 biological processes important for Brucella virulence (Fig. 8). These simple but powerful SPARQL queries demonstrate the applications of IDOBRU and IDOBRU-based SPARQL technology.

One important contribution of this paper is its first report in co-representing genes and proteins using the Ontology of Genes and Genomes (OGG) [19] and the Protein Ontology (PRO) [20]. The majority of Brucella virulence factors are proteins, which are encoded by specific genes in different Brucella strains. The practice of using IDOBRU IDs in our original IDOBRU version was not ideal since it does not support ontology reuse and integration. To address this issue, the new version of IDOBRU uses the gene and protein IDs from the OGG and $\mathrm{PRO}$, two ontologies in the OBO ontology library. The OGG ontology was recently developed to represent genes from specific organisms by reusing existing resources, primarily the NCBI Gene resource [59]. Due to the large numbers of genes available in different organisms, OGG includes different subsets, each of which represents genes from one or a few organisms. Using the OGG development strategy, we first generated the OGG Brucella subset that covers all genes of three major Brucella strains. All virulence factor genes covered in IDOBRU are from these three Brucella strains. The availability of the OGG Brucella subset allows us to retrieve and reuse the OGG terms to represent Brucella genes in IDOBRU. Similarly, specific 
Brucella proteins were generated in PRO and reused in IDOBRU. Furthermore, using two object properties (i.e., 'has_gene_template' and 'encodes'), we were able to represent the relations between genes and proteins. Since genes and proteins are two fundamental entities in biology, this study provide a demonstration on how these can be ontologically represented and interlinked.

\section{Conclusions}

In this paper, we ontologically represent various Brucellahost interactions primarily using the Brucella-macrophage interaction as a use case. A formal definition of the Brucella-host interaction was given in OWL format. After the definitions on smooth and rough Brucella are given, six subtypes of Brucella-host interactions are classified according to the Brucella phenotypes and host cell types. IDOBRU is further used as a platform to represent interactive processes including Brucella invasion, intracellular trafficking and intracellular replication at an organism level. By representing the Brucella pathogenesis mechanisms using Brucella T4SS and EryC examples, we demonstrate how to ontologically link biological processes from the organism level down to the molecular level. Description logical inference rules have also been defined to infer: 1) the interaction process between two species (i.e., a host and a pathogen); 2) the temporal relations of biological processes; 3 ) relations between gene, protein, genome, and gene mutant; and 4) a virulence factor. For this study, many new terms have been added into IDOBRU. Using SPARQL queries generated based on inference rules, out of the 269 virulence factors related to macrophage-Brucella interactions, 81 virulence factors were found to be important for Brucella intracellular replication inside macrophage. Eleven biological processes were also found important for Brucella virulence.

\section{Methods}

\section{Ontology editing}

The format of W3C standard Web Ontology Language (OWL2) (http://www.w3.org/TR/owl-guide/) was applied for IDOBRU development. The Protégé OWL ontology editor (http://protege.stanford.edu/) (versions 4.3 and 5.0 beta) was used to edit IDOBRU.

\section{Existing ontology term import}

The ontology development uses a hybrid bottom-up and top-down method as described in our original IDOBRU article [10]. For this host-Brucella interaction study, many external ontology terms from existing ontologies, including Cell Type Ontology (CL) [14], Chemical Entities of Biological Interest (ChEBI) [15], Gene Ontology (GO) [16], Protein Ontology (PRO) [20], were imported to IDOBRU using OntoFox (http://ontofox.hegroup.org/) [31].

\section{IDOBRU access and visualization}

The latest version of IDOBRU is always available at Sourceforge website: (http://svn.code.sf.net/p/idobru/code/trunk/ src/ontology/brucellosis.owl). Not that this is an unmerged OWL file, and it imports many other OWL files in the same folder. Therefore, it would be best to get all the related ontology files via SVN. IDOBRU has also been deposited in the NCBO BioPortal (http://bioportal.bioontology. org/ontologies/IDOBRU) and the Ontobee linked ontology browser system (http://www.ontobee.org/browser/index. php?o=IDOBRU). Both NCBO BioPortal and Ontobee provide interactive search and visualization features for IDOBRU exploration and analysis.

\section{OGG and PRO representation of Brucella virulence factors} The Brucella subset of the Ontology of Genes and Genomes (OGG) was generated using a method described in the OGG paper [19]. Specifically, a NCBITaxon subset was generated to include three Brucella strains using OntoFox [31]. These strains are B. abortus strain 2308, B. suis strain 1330 , and B. melitensis strain $16 \mathrm{M}$. All annotated Brucella genes in IDOBRU come from these three strains. Most of the information of all added Brucella genes encoding protein virulence factors was obtained from the manually annotated Victors database (http:// www.phidias.us/victors) in the PHDIAS resource [60]. The OGG Brucella subset was submitted to the He group RDF triple store [61]. OntoFox was then used to retrieve the Brucella genes covered in IDOBRU.

The corresponding proteins encoded by these Brucella genes are represented by Protein Ontology (PRO) [20]. OntoFox was used to extract the information of these proteins from PRO. The resulting PRO subset was then imported to IDOBRU.

\section{Queries of IDOBRU}

SPARQL scripts were developed to query IDOBRU using the IDOBRU SPARQL query web page (http://www. phidias.us/bbp/idobru/sparql/index.php) located in the Brucella Bioinformatics Portal (BBP; http://www.phidias. us/bbp) [60, 62].

\section{Implementation of inference rules}

The reasoner HermiT 1.3.8 (http://hermit-reasoner.com/) as a plugin in the Protégé OWL editor (http://protege. stanford.edu/) was used to implement the inference rules defined in this paper. The rule view editor in the Protégé OWL editor was used to edit the rules. The ontology rule view in Protégé is accessible from the Protégé menu Window $\rightarrow$ Views $\rightarrow$ Ontology views $\rightarrow$ Rules. The saved IDOBRU OWL file contains the rules in the format of OWL with SWRL codes. 


\section{Additional files}

\section{Additional file 1: Implementation of inference rules using the} Protégé platform. (PDF $383 \mathrm{~kb}$ )

Additional file 2: SPARQL query of IDOBRU for the total number of protein virulence factors in IDOBRU. (PDF $309 \mathrm{~kb}$ )

Additional file 3: SPARQL query of IDOBRU for Brucella mutants that are attenuated inside macrophages during the macrophageBrucella interactions. Since each mutant is associated with one gene and one protein, these queries also allow us extract those virulence genes and protein virulence factors that participate in various macrophage-Brucella interactions. (PDF $353 \mathrm{~kb}$ )

Additional file 4: SPARQL query of IDOBRU for Brucella protein virulence factors important for the intracellular replication of Brucella inside macrophages. (PDF $198 \mathrm{~kb}$ )

\section{Competing interests}

The authors declare that they have no competing interests.

\section{Authors' contributions}

YL: Primary IDOBRU ontology developer, SPARQL analysis, and use case testing. ZX: IDOBRU developer, SPARQL analysis, and manuscript editing. YH: IDOBRU developer, project design and management, brucellosis domain expert, and SPARQL analysis, and use case testing. The manuscript was primarily drafted by $\mathrm{YL}$ and $\mathrm{YH}$, and edited and approved for publications by all authors.

\section{Acknowledgements}

This work has been supported by grant R01Al081062 from the NIH National Institute of Allergy and Infectious Diseases (NIAID). The article-processing charge for this article was paid by a bridge fund to $\mathrm{YH}$ from the Unit for Laboratory Animal Medicine (ULAM) in the University of Michigan. We thank Dr. Salwa Ali and Mr. Jiangan Xie for contributing to the IDOBRU generation. We appreciate Drs. Darren A. Natale and Cathy H. Wu for their support on adding related Brucella proteins to PRO and their comments and editing of the manuscript.

Received: 31 December 2012 Accepted: 23 September 2015 Published online: 05 October 2015

\section{References}

1. Corbel MJ. Brucellosis: an overview. Emerg Infect Dis. 1997;3(2):213-21.

2. Pappas G, Papadimitriou P, Akritidis N, Christou L, Tsianos EV. The new global map of human brucellosis. Lancet Infect Dis. 2006;6(2):91-9.

3. Jarvis BW, Harris TH, Qureshi N, Splitter GA. Rough lipopolysaccharide from Brucella abortus and Escherichia coli differentially activates the same mitogen-activated protein kinase signaling pathways for tumor necrosis factor alpha in RAW 264.7 macrophage-like cells. Infect Immun. 2002;70(12):7165-8.

4. $\quad$ Xiang Z, Zheng W, He Y. BBP: Brucella genome annotation with literature mining and curation. BMC Bioinformatics. 2006;7(1):347

5. He Y. Analyses of Brucella pathogenesis, host immunity, and vaccine targets using systems biology and bioinformatics. Front Cell Infect Microbiol. 2012;2:2

6. Kohler S, Michaux-Charachon S, Porte F, Ramuz M, Liautard JP. What is the nature of the replicative niche of a stealthy bug named Brucella? Trends Microbiol. 2003;11(5):215-9.

7. He Y, Vemulapalli R, Zeytun A, Schurig GG. Induction of specific cytotoxic lymphocytes in mice vaccinated with Brucella abortus RB51. Infect Immun. 2001;69(9):5502-8

8. He Y, Reichow S, Ramamoorthy S, Ding X, Lathigra R, Craig JC, et al. Brucella melitensis triggers time-dependent modulation of apoptosis and downregulation of mitochondrion-associated gene expression in mouse macrophages. Infect Immun. 2006;74(9):5035-46.

9. Cowell LG, Smith B. Infectious disease ontology. In: Sintchenko V, editor. Infectious disease informatics. New York Dordrecht Heidelberg London: Springer; 2010. p. 373-95.

10. Lin Y, Xiang Z, He Y. Brucellosis Ontology (IDOBRU) as an extension of the Infectious Disease Ontology. J Biomed Semant. 2011;2(1):9.
11. Smith B, Ashburner M, Rosse C, Bard J, Bug W, Ceusters W, et al. The OBO Foundry: coordinated evolution of ontologies to support biomedical data integration. Nat Biotechnol. 2007;25(11):1251-5.

12. Grenon P. Spatio-temporality in basic formal ontology. In: Grenon P, editor. IFOMIS reports. Leipzig: Institute for Formal Ontology and Medical Information Science at the Faculty of Medicine of the University of Leipzig; 2003. p. 89.

13. Grenon P, Smith B. SNAP and SPAN: towards dynamic spatial ontology. Spat Cogn Comput. 2004;4(1):69-103.

14. Diehl AD, Augustine AD, Blake JA, Cowell LG, Gold ES, Gondre-Lewis TA, et al. Hematopoietic cell types: prototype for a revised cell ontology. J Biomed Inform. 2011;44(1):75-9.

15. Hastings J, de Matos P, Dekker A, Ennis M, Harsha B, Kale N, et al. The ChEBI reference database and ontology for biologically relevant chemistry: enhancements for 2013. Nucleic Acids Res. 2013;41(Database issue):D456-63.

16. Ashburner M, Ball CA, Blake JA, Botstein D, Butler H, Cherry JM, et al. Gene ontology: tool for the unification of biology. The Gene Ontology Consortium. Nat Genet. 2000;25(1):25-9.

17. Information Artifact Ontology. http://code.google.com/p/information-artifactontology/, accessed on October 2, 2015.

18. Brinkman RR, Courtot M, Derom D, Fostel JM, He Y, Lord P, et al. Modeling biomedical experimental processes with OBI. J Biomed Semant. 2010;1 Suppl 1:S7.

19. He Y, Liu Y, Zhao B. OGG: a biological ontology for representing genes and genomes in specific organisms. In: The 2014 International Conference on Biomedical Ontologies (ICBO 2014): October 8-9, 2014 2014; Houston, TX, USA. CEUR Workshop Proceedings. 2014. p. 13-20.

20. Natale DA, Arighi CN, Blake JA, Bult CJ, Christie KR, Cowart J, et al. Protein Ontology: a controlled structured network of protein entities. Nucleic Acids Res. 2014;42(Database issue):D415-21.

21. Smith B, Ceusters W, Klagges B, Kohler J, Kumar A, Lomax J, et al. Relations in biomedical ontologies. Genome Biol. 2005;6(5):R46

22. O'Connor M, Knublauch H, Tu S, Grosof B, Dean M, Grosso W, et al. Supporting rule system interoperability on the Semantic Web with SWRL. Lect Notes Comput Sci. 2005;3729:974-86.

23. SPARQL query language for RDF. http://www.w3.org/TR/rdf-sparql-query/, accessed on October 2, 2015.

24. Moriyon I, Lopez-Goni I. Structure and properties of the outer membranes of Brucella abortus and Brucella melitensis. Int Microbiol. 1998;1(1):19-26.

25. Chen F, Ding X, Ding Y, Xiang Z, Li X, Ghosh D, et al. Proinflammatory caspase-2-mediated macrophage cell death induced by a rough attenuated Brucella suis strain. Infect Immun. 2011;79(6):2460-9.

26. Ceusters W, Elkin P, Smith B. Negative findings in electronic health records and biomedical ontologies: a realist approach. Int J Med Inform. 2007;76 Suppl 3:S326-33.

27. Hoehndorf R, Oellrich A, Dumontier M, Kelso J, Rebholz-Schuhmann D, Herre H. Relations as patterns: bridging the gap between $\mathrm{OBO}$ and $\mathrm{OWL}$. BMC Bioinformatics. 2010;11:441.

28. Roop 2nd RM, Gaines JM, Anderson ES, Caswell CC, Martin DW. Survival of the fittest: how Brucella strains adapt to their intracellular niche in the host. Med Microbiol Immunol. 2009;198(4):221-38.

29. Briones $G$, Inon de lannino N, Roset M, Vigliocco A, Paulo PS, Ugalde RA. Brucella abortus cyclic beta-1,2-glucan mutants have reduced virulence in mice and are defective in intracellular replication in HeLa cells. Infect Immun. 2001;69(7):4528-35.

30. Sarntivijai $S$, Lin $Y$, Xiang Z, Meehan TF, Diehl AD, Vempati UD, et al. CLO: the cell line ontology. J Biomed Semant. 2014;5:37.

31. Xiang Z, Courtot M, Brinkman RR, Ruttenberg A, He Y. OntoFox: web-based support for ontology reuse. BMC Res Notes. 2010;3(175):1-12.

32. Baldwin $\mathrm{CL}$, Winter AJ. Macrophages and Brucella. Immunol Ser. 1994:60:363-80

33. Pei J, Ficht TA. Brucella abortus rough mutants are cytopathic for macrophages in culture. Infect Immun. 2004;72(1):440-50.

34. Chen F, He Y. Caspase-2 mediated apoptotic and necrotic murine macrophage cell death induced by rough Brucella abortus. PLoS One. 2009;4(8):e6830.

35. Bronner D, O'Riordan M, He Y. Caspase-2 mediates a Brucella abortus RB51induced hybrid cell death having features of apoptosis and pyroptosis. Front Cell Infect Microbiol. 2013;3:38. doi:10.3389/fcimb.2013.00083.

36. Rittig MG, Kaufmann A, Robins A, Shaw B, Sprenger H, Gemsa D, et al. Smooth and rough lipopolysaccharide phenotypes of Brucella induce different intracellular trafficking and cytokine/chemokine release in human monocytes. J Leukoc Biol. 2003;21:21. 
37. Kalde M, Moreno E, Gorvel JP. Brucella. In: Schaible UE, Haas A, editors. Intracellular niches of microbes: a pathogens guide through the host cell. Wiley Online Library. 2009.

38. Pei J, Wu Q, Kahl-McDonagh M, Ficht TA. Cytotoxicity in macrophages infected with rough Brucella mutants is type IV secretion system dependent. Infect Immun. 2008;76(1):30-7.

39. O'Callaghan D, Cazevieille C, Allardet-Servent A, Boschiroli ML, Bourg G, Foulongne $\mathrm{V}$, et al. A homologue of the Agrobacterium tumefaciens VirB and Bordetella pertussis PtI type IV secretion systems is essential for intracellular survival of Brucella suis. Mol Microbiol. 1999;33(6):1210-20.

40. Boschiroli ML, Ouahrani-Bettache S, Foulongne V, Michaux-Charachon S, Bourg G, Allardet-Servent A, et al. Type IV secretion and Brucella virulence. Vet Microbiol. 2002;90(1-4):341-8.

41. de Jong MF, Sun $Y H$, den Hartigh AB, van Dijl JM, Tsolis RM. Identification of VceA and VceC, two members of the VjbR regulon that are translocated into macrophages by the Brucella type IV secretion system. Mol Microbiol. 2008;70(6):1378-96.

42. Arocena GM, Sieira R, Comerci DJ, Ugalde RA. Identification of the quorumsensing target DNA sequence and $\mathrm{N}$-Acyl homoserine lactone responsiveness of the Brucella abortus virB promoter. J Bacteriol. 2010;192(13):3434-40.

43. de Barsy M, Jamet A, Filopon D, Nicolas C, Laloux G, Rual JF, et al. Identification of a Brucella spp. secreted effector specifically interacting with human small GTPase Rab2. Cell Microbiol. 2011;13(7):1044-58.

44. Dong C, Wu G. Regulation of anterograde transport of adrenergic and angiotensin II receptors by Rab2 and Rab6 GTPases. Cell Signal. 2007;19(11):2388-99.

45. de Bolle X, Letesson JJ, Gorvel JP. Small GTPases and Brucella entry into the endoplasmic reticulum. Biochem Soc Trans. 2012;40(6):1348-52.

46. Burkhardt S, Jimenez de Bagues MP, Liautard JP, Kohler S. Analysis of the behavior of eryC mutants of Brucella suis attenuated in macrophages. Infect Immun. 2005;73(10):6782-90.

47. Sangari FJ, Aguero J, Garcia-Lobo JM. The genes for erythritol catabolism are organized as an inducible operon in Brucella abortus. Microbiology. 2000;146(Pt 2):487-95.

48. Crasta OR, Folkerts O, Fei Z, Mane SP, Evans C, Martino-Catt S, et al. Genome sequence of Brucella abortus vaccine strain S19 compared to virulent strains yields candidate virulence genes. PLoS One. 2008;3(5):e2193.

49. Hill DP, Berardini TZ, Howe DG, Van Auken KM. Representing ontogeny through ontology: a developmental biologist's guide to the gene ontology. Mol Reprod Dev. 2010;77(4):314-29.

50. Schurig GG, Roop RMD, Bagchi T, Boyle S, Buhrman D, Sriranganathan N. Biological properties of RB51; a stable rough strain of Brucella abortus. Vet Microbiol. 1991;28(2):171-88.

51. Iguchi K, Sakurai F, Tomita K, Katayama K, Yamaguchi T, Kawabata K, et al. Efficient antitumor effects of carrier cells loaded with a fiber-substituted conditionally replicating adenovirus on CAR-negative tumor cells. Cancer Gene Ther. 2011;9(2):118-25.

52. Bandara AB, Poff-Reichow SA, Nikolich M, Hoover DL, Sriranganathan N, Schurig GG, et al. Simultaneous expression of homologous and heterologous antigens in rough, attenuated Brucella melitensis. Microbes Infect. 2009;11(3):424-8.

53. He Y, Cowell L, Diehl AD, Mobley HL, Peters B, Ruttenberg A, et al. VO: vaccine ontology. In: The 1st International Conference on Biomedical Ontology (ICBO-2009): July 24-26 2009; Buffalo, NY, USA. Nature Proceedings. 2009. http://precedings.nature.com/documents/3552/version/1, accessed on October 2, 2015

54. Ozgur A, Xiang Z, Radev DR, He Y. Mining of vaccine-associated IFN-gamma gene interaction networks using the Vaccine Ontology. J Biomed Semant. 2011;2 Suppl 2:S8.

55. Lindeberg M, Biehl BS, Glasner JD, Perna NT, Collmer A, Collmer CW. Gene Ontology annotation highlights shared and divergent pathogenic strategies of type III effector proteins deployed by the plant pathogen Pseudomonas syringae pv tomato DC3000 and animal pathogenic Escherichia coli strains. BMC Microbiol. 2009;9 Suppl 1:S4

56. Topalis P, Mitraka E, Bujila I, Deligianni E, Dialynas E, Siden-Kiamos I, et al. IDOMAL: an ontology for malaria. Malar J. 2010;9:230.

57. Gorvel JP, Moreno E. Brucella intracellular life: from invasion to intracellular replication. Vet Microbiol. 2002;90(1-4):281-97.

58. Harris S, Seaborne A. SPARQL 1.1 Query Language, W3C Recommendation 21 March 2013. 2013. URL: http://www.w3.org/TR/sparql11-query/, accessed on August 14, 2013.
59. NCBI Gene, a portal to gene-centered information from different sources ftp://ftp.ncbi.nih.gov/pub/factsheets/Factsheet_Gene.pdf, accessed on October 2, 2015.

60. Xiang Z, Tian Y, He Y. PHIDIAS: a pathogen-host interaction data integration and analysis system. Genome Biol. 2007;8(7):R150.

61. Xiang Z, Mungall C, Ruttenberg A, He Y. Ontobee: A linked data server and browser for ontology terms. In: The 2nd International Conference on Biomedical Ontologies (ICBO): 2011; Buffalo, NY, USA. CEUR Workshop Proceedings. 2013. p. 279-81. http://ceur-ws.org/Vol-833/paper48.pdf, accessed on October 2, 2015.

62. Xiang Z, Zheng W, He Y. BBP: Brucella genome annotation with literature mining and curation. BMC Bioinformatics. 2006;7:347.

\section{Submit your next manuscript to BioMed Central and take full advantage of:}

- Convenient online submission

- Thorough peer review

- No space constraints or color figure charges

- Immediate publication on acceptance

- Inclusion in PubMed, CAS, Scopus and Google Scholar

- Research which is freely available for redistribution 